\title{
Statistical analysis of the electronic structure of single-wall carbon nanotubes
}

\author{
I. Wirth, S. Eisebitt, G. Kann, and W. Eberhardt \\ Institut für Festkörperforschung, Forschungszentrum Jülich, D-52425 Jülich, Germany
}

(Received 22 June 1999)

\begin{abstract}
The electronic structure of single-wall nanotubes in intact, undissolved "buckypaper" has been studied using scanning tunneling microscopy (STM) and scanning tunneling spectroscopy (STS) at $23 \mathrm{~K}$. STM topography shows, that single-wall carbon nanotubes (SWNT's) form microbundles that in turn aggregate in ropes. STS allows us to distinguish between room temperature metallic and wide-gap SWNT's. We find a distribution ratio of $(49 \pm 7) \%:(51 \pm 7) \%$, respectively. A statistical analysis of the observed band gaps and metallic plateaus within these groups is carried out and used to determine the diameter distribution of the SWNT's in the buckypaper.
\end{abstract}

Single-wall carbon nanotubes (SWNT's) are a relativeley new class of carbon materials. They can be thought of as a graphene layer rolled up along one of its twodimensional (2D) lattice vectors $\mathbf{R}=n_{1} \mathbf{R}_{1}+n_{2} \mathbf{R}_{2}$ to form a tube. With $\mathbf{R}_{1}$ and $\mathbf{R}_{2}$ being primitive lattice vectors of the graphene lattice, the SWNT's are characterized by the coefficients $\left(n_{1}, n_{2}\right)$. The tube diameters $D=|\mathbf{R}| / \pi$ $=\left(a_{c c} / \pi\right) \sqrt{3\left(n_{1}^{2}+n_{2}^{2}+n_{1} n_{2}\right)} \quad\left(a_{c c}\right.$ : C $-\mathrm{C}$ bond distance $)$ are in the range of a few nm while the SWNT's can be up to several $\mu \mathrm{m}$ long. ${ }^{1}$

While carbon nanotubes are interesting for a variety of reasons, e.g., their mechanical properties, ${ }^{2}$ single-wall carbon nanotubes are particularly interesting because of their electronic structure, which strongly depends on the chirality vector $\mathbf{R}$ defining the type of nanotube. This is due to the effect that the electronic states with wave vectors parallel to $\mathbf{R}$ have to satisfy periodic boundary conditions and hence experience quantum confinement.

To a certain degree of approximation, the electronic structure of SWNT's can be deduced from the graphene band structure by investigating which electronic states in graphene are allowed in SWNT's, given the boundary conditions in a specific tube. Since the graphite bands are crossing $E_{F}$ at the $K$ point at the corner of the Brillouin zone, only those nanotubes whose band structure contains this point are metallic. It turns out that a $\left(n_{1}, n_{2}\right)$ SWNT is metallic if $\left(n_{1}-n_{2}\right)$ is an integer multiple of 3 and semiconducting otherwise. Beyond the graphene approximation, i.e., including curvatureinduced hybridization effects, it turns out that only ( $n_{1}$ $=n_{2}$ ) and equivalent SWNT's ("armchair" type) are strictly metallic, whereas small band gaps form in all other SWNT's that have been classified as metallic in the graphene approximation. ${ }^{1,3,4}$ These SWNT's are referred to as narrowgap SWNT's in contrast to the wide gap SWNT's with $\left(n_{1}\right.$ $\left.-n_{2}\right) \neq 3 q$. The size of the narrow gap decreases like the inverse square of the SWNT radius. In the literature, metallic and near gap SWNT's are often not distinguished and the union of both sets is then (incorrectly) referred to as "metallic SWNT's." In this paper we will use the term "roomtemperature metallic" (RT-metallic) to refer to SWNT's that are either metallic or near gap semiconductors.

In this paper we determine the distribution of SWNT's in a specific sample with regard to their electronic structure.
Most spectroscopic techniques that are directly sensitive to the electronic structure probe a large number of individual nanotubes in one experiment. While such experiments can demonstrate the existence of metallic (or narrow gap or wide gap) nanotubes in a given nanotube ensemble, they cannot quantify how much nanotubes of which type are in the sample, as this would require reference spectra of all types of pure $\left(n_{1}, n_{2}\right)$ SWNT's. ${ }^{4,5}$ Most geometry-sensitive techniques such as transmission electron microscopy (TEM) do not have direct access to the electronic structure. Only if the resolution is sufficient to resolve the specific type $\left(n_{1}, n_{2}\right)$ of SWNT's, can one try to deduce electronic structure information from the geometry information using theoretical models. These models typically assume ideal, single-wall nanotubes of infinite length, without twist or bend and without interactions to other SWNT's and are hence of limited applicability.

These difficulties can be overcome with a local spectroscopic technique, such as scanning tunneling spectroscopy (STS). The electronic structure of individual nanotubes in a sample can directly be investigated. A study of a statistically signifcant number of nanotubes at random positions in a given sample will thus enable us to determine the fraction of, e.g., metallic nanotubes in the sample.

For this study, we have chosen a nanotube sample that was prepared by pulsed laser vaporization (PLV) and purified as described in Ref. 6 (4" oven material). The overall production process including the purification has been shown to produce a material with a high degree of SWNT's, distributed over a narrow diameter range. ${ }^{6}$ The final product is a paperlike felt, called "buckypaper."

The investigations were carried out in a UHV STM chamber with a base pressure less than $1 \times 10^{-10}$ Torr. The buckypaper was cleaned after exposure to air by heating to $1200^{\circ} \mathrm{C}$ in UHV.

STS and imaging were performed with an Omicron VT STM using an etched tungsten tip. The sample was cooled by liquid helium to a temperature of $23 \mathrm{~K}$. Both free-standing buckypaper and buckypaper partially immersed in silver epoxy were investigated. In both cases, the buckpaper was neither disentangled nor dissolved and hence the distribution of SWNT's is unchanged from the freshly purified sample. We will first present results on the topography of the buckypaper before we discuss the STS results. 


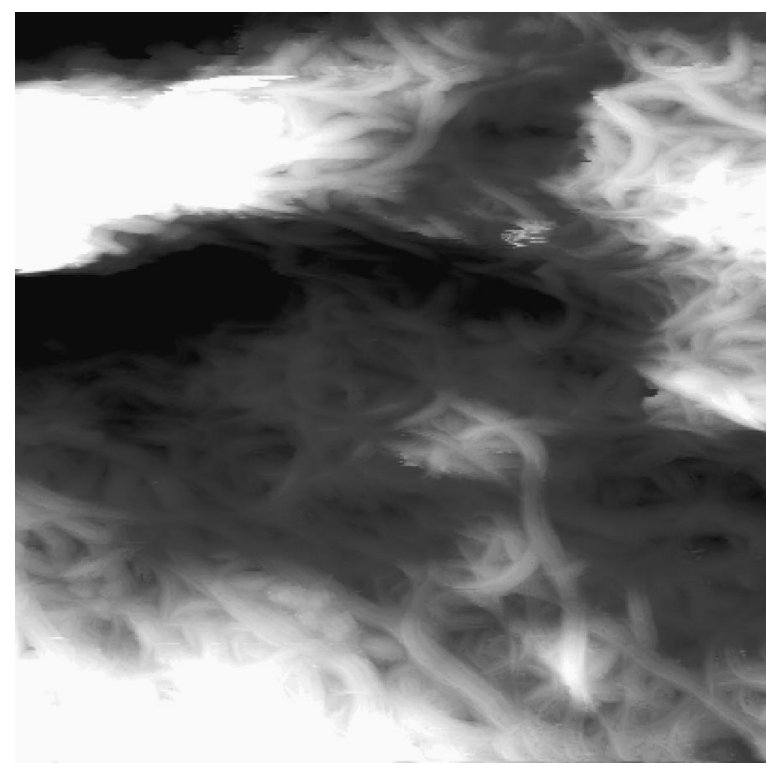

FIG. 1. STM overview image of the free-standing sheet of buckypaper $\left(2000 \times 2000 \mathrm{~nm}^{2}\right)$.

An overview STM image of a free-standing sheet of buckypaper is shown in Fig. 1. Clearly visible are ropes with diameters between 70 and $120 \mathrm{~nm}$ which are interwoven, forming the felt. These ropes are not individual SWNT's, but metastructures with SWNT's as the individual building blocks. Such ropes have been observed for nanotubes produced by different techniques. Depending on the growth conditions, the organization of the individual nanotubes into larger structures can be different. ${ }^{7-10}$

In Figs. 1 and 2, the organization of SWNT's into larger structures within our buckypaper sample can be seen. According to the measurements in Ref. 6, the individual SWNT's in our type of sample are $1.0-1.5 \mathrm{~nm}$ in diameter. We have reproduced the TEM diameter distribution from Ref. 6, as shown in Fig. 3. The individual SWNT's form

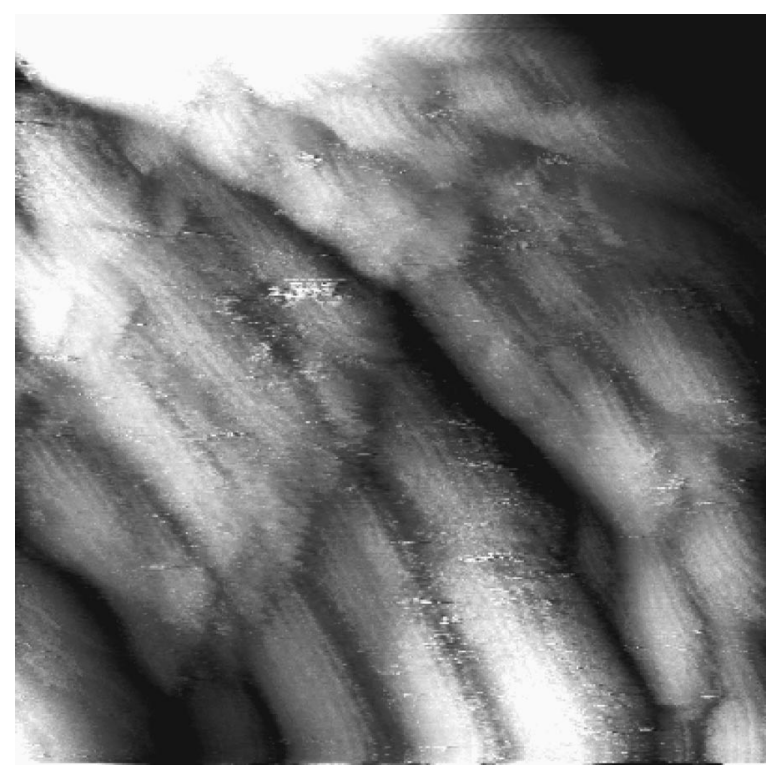

FIG. 2. STM image: parallel SWNT's (fine diagonal lines) form microbundles, which in turn form the ropes visible in Fig. 1 (250 $\times 250 \mathrm{~nm}^{2}$ ).

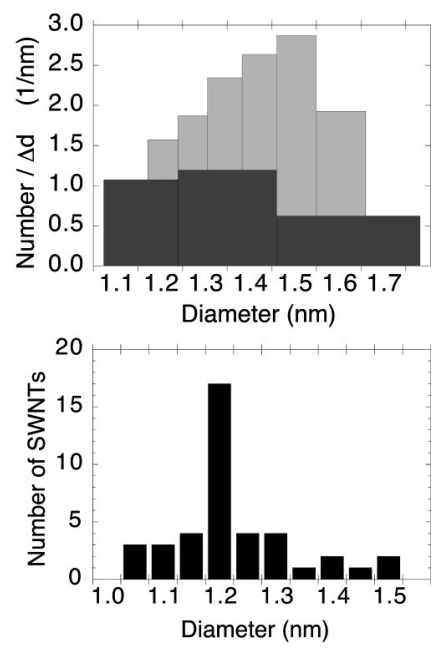

FIG. 3. Top: Diameter distribution of SWNT's calculated from the band-gap and/or metallic plateau distributions assuming ideal SWNT's. Dark histogram bars indicate wide gap SWNT's, gray bars refer to RT-metallic SWNT's. Bottom: SWNT diameter distribution in buckypaper as determined by transmission electron microscopy in Ref. 7. The diameter scales were established using $V_{p p \pi}=2.7 \mathrm{eV}$. According to Eqs. (1) and (2), the diameter depends linearly on $V_{p p \pi}$ for a given $\delta E$. The uncertainty of the size of $V_{p p \pi}$ (Ref. 17) has thus to be taken into account.

microbundles of about $30 \mathrm{~nm}$ in diameter. In the microbundles, the SWNT's are organized in a parallel fashion (Fig. 2). A strikingly similar arrangement has been reported for nanotubes generated in a carbon arc plasma. ${ }^{7}$ The microbundles aggregate to ropes of about 70-100-nm diameter (Fig. 2). On the length scales from the SWNT's to the ropes we observe no significant amounts of globular structures due to carbon in other forms than SWNT's, in agreement with other studies of the buckypaper before and after purification. ${ }^{6}$

STM images on the intact buckypaper could only be obtained with voltage and/or current parameters corresponding to relatively large tip-sample separations. For short separations, the image becomes unstable due to tip-induced motions of the nanotubes. For this reason, atomic resolution could not be consistently achieved on the buckypaper. So far, atomic resolution on SWNT's has only been reported for single tubes ${ }^{11,12}$ or small ropes ${ }^{8}$ which have been prepared on metallic substrates. The investigation of the electronic structure of the SWNT's has been carried out by STS. I $(V)$ curves were recorded at 114 randomly choosen positions on different SWNT ropes.

With the sample being a network of not necessarilly well conducting ropes, one has to address the question of which electronic properties are probed by STS. It could be possible that internal tunnel junctions, e.g., between crossing ropes, dominate the appearance of the $I(V)$ curve. In such a case, the $I(V)$ curve could practically not be used to extract information on the electronic structure of the SWNT adjacent to the tip.

This concern, however, turns out to be unsubstantiated. $I(V)$ curves recorded with the tip in contact with the buckypaper at various positions all show perfectly metallic behavior. We conclude that the conductivity through the network is always high enough to short out internal tunnel junctions. This observation is in line with (a) the observed conductivity 


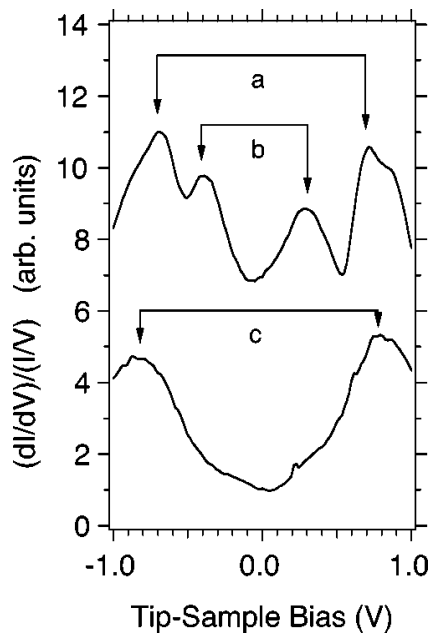

FIG. 4. Example of STS data recorded at $23 \mathrm{~K}$ on the buckypaper. Shown is the normalized conductance $(d I / d V) /(I / V)$ as a function of the tip-sample bias. Regularly spaced pairs of singularities (arrows) are visible. Top: the pairs of singularities observed with a spacing of $1.4 \mathrm{eV}$ (a) and $0.7 \mathrm{eV}$ (b) indicate a wide-gap behavior of this SWNT. Bottom: the smallest singularity spacing observed is $1.6 \mathrm{eV}$ (c), indicating that the SWNT probed is RTmetallic.

value of less than $0.001 \Omega \mathrm{cm},{ }^{6}$ (b) the fact that we find about 1/2 RT-metallic SWNT's in the sample (see below), and (c) on the order of ten rope crossings per micrometer from the inspection of STM overview images.

In order to study the electronic structure of the SWNT's we investigate the shape of the normalized differential conductivity, $(d I / d V) /(I / V)$, which represents in good approximation the density of states (DOS) of the sample. ${ }^{13}$. In agreement with the theoretical expectations, ${ }^{1}$ we observe pronounced peaks in the DOS corresponding to the typical singularities in the DOS for low-dimensional systems. The peaks are visible in both $(d I / d V)$ and $(d I / d V) /(I / V)$ plots of the data.

In order to classify the SWNT's into the categories RTmetallic versus wide gap, we determine the energy positions of these singularities. For wide gap SWNT's, the energy spacing $\Delta E_{w g}^{m}$ of the first two pairs of singularities (symmetrically around $V=0$ ) is

$$
\Delta E_{w g}^{m}=\frac{2 V_{p p \pi} a_{c c}}{D} m, \quad m=1,2
$$

with $V_{p p \pi}$ being the next-nearest-neighbor interaction. ${ }^{3,14}$ For the case of wide gap SWNT's, $\Delta E_{w g}^{1}$ is identical to the band gap. In contrast to a direct determination of the band gap by STS at $23 \mathrm{~K}$ and a relatively large tip-sample distance, Eq. (1) allows a precise determination of the band gap via the singularities, as the peak positions can be determined accurately and two pairs of singularities can be used. An example is shown in Fig. 4. We estimate the overall error in the determination of the band gap to be about $0.1 \mathrm{eV}$.

Metallic SWNT's do not have a band gap; however, the region between the two innermost singularities $(m=1)$ is a metallic plateau (i.e., a region of significantly lower DOS than at adjacent energies), which is characteristic for the
SWNT in question. Both metallic and near gap SWNT's exhibit the same dependence of $\Delta E^{m}$ on the SWNT radius, ${ }^{3,14}$

$$
\Delta E_{m e t, n g}^{1}=\frac{6 V_{p p \pi} a_{c c}}{D} .
$$

As a curvature effect, the formation of a band gap in the near gap SWNT's is not described by Eq. 2. According to Refs. 3 and 14, the approximations used to derive Eqs. (1) and (2) introduce only small errors in the determination of $\Delta E$ for bias voltages up to about $\pm 1 \mathrm{~V}(D<2 \mathrm{~nm})$, as compared with the error derived from the experimental STS resolution. In our data analysis, Eq. (2) was used to determine $\Delta E_{\text {met,ng }}$; all observed singularities were used in the case of wide gap SWNT's as Eq. (1) $(m=1,2)$ was used to determine $\Delta E_{w g}$. We would like to emphazise that we do not use the connection between $D$ and $\Delta E$ in Eqs. (1) and (2) in our analysis, as we obtain the electronic structure information directly. We only take advantage of the fact that we can increase the accuracy of the $\Delta E$ determination by using $m$ $=1,2$. The above formulas have been derived for isolated SWNT's of "ideal" shape.

For the same SWNT diameter, $\Delta E^{m}$ is three times as large for wide gap SWNT's than for metallic and near gap SWNT's. For our buckypaper sample this fact allows us to unambiguously distinguish between metallic and near gap SWNT's on one hand and wide gap SWNT's on the other on the basis of the measured $\Delta E^{m}$. For a wide gap SWNT to have the same $\Delta E^{m}$ as a metallic or near gap SWNT, the diameter of the former SWNT has to be one-third of the diameter of the latter. However, the SWNT diameter distribution in our sample is narrow and does not span a factor of 3, as can be seen in Fig. 3. Consequently, a distinction between RT-metallic and wide gap SWNT's on the basis of $\Delta E^{m}$ is readily accomplished. We find $49 \pm 7 \%$ RT-metallic SWNT's versus $51 \pm 7 \%$ wide gap SWNT's in the buckypaper sample.

A distinction between metallic and near gap SWNT's is not possible on this basis. Although we sometimes observe STS features close to zero bias which might be associated with the existence of a narrow gap, theses features are too smeared out to allow for an unambiguous distinction between metallic and near gap SWNT's in the present experiment. The width of the spectral features may in part be due to SWNT bend and twist or intrarope interactions.

For the set of all theoretically possible $\left(n_{1}, n_{2}\right)$ SWNT's, one would expect a ratio of $1 / 3$ RT-metallic vs $2 / 3$ wide gap SWNT's. If we only consider such $\left(n_{1}, n_{2}\right)$ SWNT's which will result in SWNT's contained in the diameter distribution shown in Fig. 3 and assume that all suitable $\left(n_{1}, n_{2}\right)$ SWNT's were to contribute equally to a given SWNT diameter, one would expect a ratio of $39 \pm 3 \%$ RT-metallic vs $61 \pm 3 \%$ wide gap SWNT's. This expectation is based on an ensemble of ideal SWNT's and the rules outlined in the introduction of this paper. In comparison to these values, our experimental findings of $49 \pm 7 \%$ RT-metallic SWNT's versus $51 \pm 7 \%$ wide gap SWNT's suggest that metallic SWNT's $\left(n_{1}, n_{2}\right)$ are preferentially produced in the PLVpurification process, although the error margins are relatively large. "Preferential production" is meant in the following way: within a given diameter interval, we find more metallic 


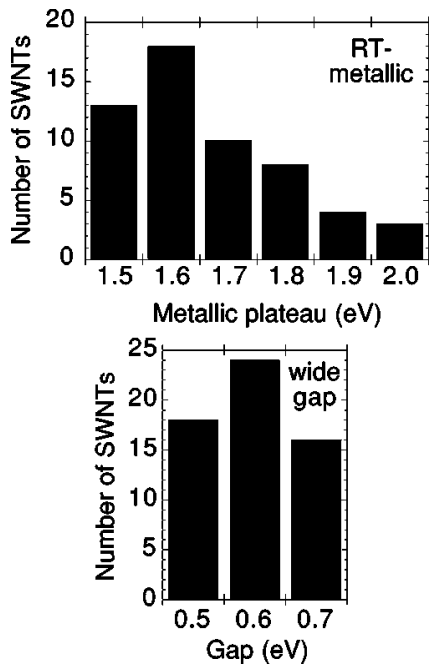

FIG. 5. Top: Metallic plateau $\left(\Delta E_{m e t, n g}\right)$ distribution within the RT-metallic SWNT's in the buckypaper sample. Bottom: Band-gap distribution within the wide gap SWNT's in the buckypaper sample.

tubes than one would expect, if all $\left(n_{1}, n_{2}\right)$ SWNT's with diameters in this intervall would contribute equally to the distribution. ${ }^{15}$

The detailed distributions of band gaps or metallic plateaus within the RT-metallic and wide gap SWNT's in our buckypaper sample are presented in the histograms in Fig. 5. We want to emphasize that the statistical data on the band gaps and metallic plateaus presented in Fig. 5 are not influenced by any theoretical assumptions on the SWNT's. STS is a direct probe of the electronic structure. This is important, as the SWNT's in buckypaper cannot necessarily be described as "ideal": The SWNT's are part of microbundles and ropes, and intrarope or especially intrabundle interactions could be important. Furthermore, the SWNT's are finite and and can be bent or twisted. Twist of SWNT's in larger aggregates has been reported to significantly alter the electronic structure. ${ }^{8,16}$ Any technique that aims to deduce the electronic structure from geometric properties, such as the diameter or the chirality of the SWNT's, will have to take such effects into account.
We expect the largest effects on the relation between geometric and electronic structure to stem from SWNT twist and intrabundle interactions. In order to investigate the deviation of the behavior from Eqs. (1) and (2) in the buckypaper sample, we have used these relations to calculate a SWNT diameter distribution from our electronic structure measurements. For our calculations, we use $V_{p p \pi}=2.7 \mathrm{eV}$ and $a_{c c}$ $=0.142 \mathrm{~nm} .{ }^{3,17}$ The resulting diameter distribution is compared to the TEM results in Fig. 3. The diameter scale depends linearly on $V_{p p \pi} . V_{p p \pi}$ values of $2.5-2.9 \mathrm{eV}$ have been reported in the literature. ${ }^{17}$ Within the error given by this $V_{p p \pi}$ interval, we observe a reasonable agreement between the diameter distributions in Fig. 3. Furthermore we observe a smeared out distribution as compared to the TEM results. This discrepancy can only partly be explained by experimental uncertainties and illustrates the limitations of Eqs. (1) and (2) for the deduction of electronic structure information of the basis of geometric structure information or vice versa.

In conclusion, we have presented results on both the topography and the electronic structure of SWNT's in buckypaper. The SWNT's were found to form microbundles of about 30-nm diameter which in turn aggregate in ropes. The ropes or metastructures composed of ropes form the "felt" of the buckypaper. By STS at $23 \mathrm{~K}$, we have directly investigated the electronic structure of the SWNT's at 114 randomly chosen points in the intact buckypaper. The distribution of band gaps and/or metallic plateaus in the SWNT's has been measured. We find RT-metallic and wide gap SWNT's with a ratio of $(49 \pm 7 \%: 51 \pm 7 \%)$, respectively, suggesting that the production of RT-metallic SWNT's is somewhat favored in the PLV-purification process. Statistical STS experiments as the one presented here allow us to quantitatively investigate the electronic structure in differently prepared samples and will be important for the optimization of the various SWNT production processes with respect to the electronic properties of the material produced.

\section{ACKNOWLEDGMENTS}

We thank J. E. Fischer and the authors of Ref. 6 for the technical help.
${ }^{1}$ M. S. Dresselhaus, G. Dresselhaus, and P. Eklund, Science of Fullerenes and Carbon Nanotubes (Academic Press, San Diego, 1996).

${ }^{2}$ T. W. Ebbesen, Phys. Today 49 (6), 26 (1996).

${ }^{3}$ J. W. Mintmire and C. T. White, Phys. Rev. Lett. 81, 2506 (1998).

${ }^{4}$ S. Eisebitt, A. Karl, W. Eberhardt, J. E. Fischer, C. Sathe, A. Agui, and J. Nordgren, Appl. Phys. A: Mater. Sci. Process. 67, 89 (1998)

${ }^{5}$ M. Knupfer, T. Pichler, M. S. Golden, J. Fink, A. Rinzler, and R. E. Smalley, Carbon 37, 733 (1999).

${ }^{6}$ A. G. Rinzler, J. Liu, H. Dai, P. Nikolaev, C. B. Huffman, F. J. Rodriguez-Macías, P. J. Boul, A. H. Lu, D. Heymann, D. T. Colbert, R. S. Lee, J. E. Fischer, A. M. Rao, P. C. Eklund, and R. E. Smalley, Appl. Phys. A: Mater. Sci. Process. 67, 29 (1998)
${ }^{7}$ T. W. Ebbesen, H. Hiura, J. Fujita, Y. Ochiai, S. Matsui, and K. Tanigaki, Chem. Phys. Lett. 209, 83 (1993).

${ }^{8}$ W. Clauss, D. J. Bergeron, and A. T. Johnson, Phys. Rev. B 58, R4266 (1998).

${ }^{9}$ J. Lefebvre, R. Antonow, and A. T. Johnson, Appl. Phys. A: Mater. Sci. Process. 67, 71 (1998).

${ }^{10}$ A. Fonseca, K. Hernadi, P. Piedigrosso, J.-F. Colomer, K. Mukhopadhyay, R. Doome, S. Lazarescu, L. P. Biro, Ph. Lambin, P. A. Thiri, D. Bernaerts, and J. B. Nagy, Appl. Phys. A: Mater. Sci. Process. 67, 11 (1998).

${ }^{11}$ J. W. G. Wildöer, L. C. Venema, A. G. Rinzler, R. E. Smalley, and C. Dekker, Nature (London) 391, 59 (1998).

${ }^{12}$ T. W. Odom, J.-L. Huang, P. Kim, and C. M. Lieber, Nature (London) 391, 62 (1998); T. W. Odom, J.-L. Huang, P. Kim, M. Ouyang, and C. M. Lieber, J. Mater. Res. 13, 2380 (1998).

${ }^{13}$ J. A. Stroscio, R. M. Feenstra, and A. P. Fein, Phys. Rev. Lett. 57, 
2579 (1986); R. M. Feenstra, J. A. Stroscio, and A. P. Fein, Surf. Sci. 181, 295 (1987).

${ }^{14}$ J.-C. Charlier and Ph. Lambin, Phys. Rev. B 57, 15037 (1998).

${ }^{15}$ It makes sense to normalize to specific diameter intervals, as in an experimental setup there must always be a preferential production of some SWNT's as compared to the theoretically possible $\left(n_{1}, n_{2}\right)$ spectrum.

${ }^{16}$ C. L. Kane and E. J. Mele, Phys. Rev. Lett. 78, 1932 (1997).

${ }^{17}$ For single SWNT's on a $\mathrm{Au}(111)$ substrate, STS/STM experi- ments correlating the electronic and geometric structure indicate the validity of Eq. (1) with a $V_{p p \pi}$ of $2.7 \mathrm{eV}$ (Ref. 11), while other experiments (Ref. 12) and calculations (Ref. 1) suggest a $V_{p p \pi}$ close to $2.5 \mathrm{eV}$. Raman experiments indicate $V_{p p \pi}$ up to $2.95 \mathrm{eV}$ (Ref. 18).

${ }^{18}$ M. A. Pimenta, A. Marucci, S. A. Empedocles, M. G. Bawendi, E. B. Hanlon, A. M. Rao, P. C. Eklund, R. E. Smalley, G. Dresselhaus, and M. S. Dresselhaus, Phys. Rev. B 58, 16016 (1998). 